\title{
RESULTADOS DE LA APLICACIÓN DE TRES GUÍAS NACIONALES PARA PREVENIR LA TRANSMISIÓN VERTICAL DEL VIH EN EL INSTITUTO NACIONAL MATERNO PERINATAL. LIMA, PERÚ
}

\author{
Carlos Velásquez $z^{1, a}$
}

\begin{abstract}
RESUMEN
Se realiza un análisis retrospectivo de tres periodos sucesivos entre los años 1996 al 2009, para evaluar el impacto de la aplicación de tres guías nacionales para la prevención de la transmisión vertical del VIH. Se incluyeron 275 nacimientos en los 13 años de estudio. Se encontraron diferencias significativas en el porcentaje de casos de VIH entre los niños expuestos al virus en los tres periodos: $15 \%$ durante el periodo en el cual solo se administraba zidovudina (AZT) a la gestante; $6,4 \%$ durante el segundo (administración de AZT a la gestante sin criterios de inicios de TARGA, y TARGA a las que tenían criterios para este tratamiento), y $4 \%$ durante el tercer periodo en el cual se aplicó TARGA a todas las gestantes con infección por VIH. El $95 \%$ de las gestantes culminaron el embarazo por cesárea y el $100 \%$ de niños recibió fórmula maternizada. Los cambios realizados en las guías nacionales han producido un impacto favorable en la disminución de nacimientos de niños infectados por el VIH en el Instituto Nacional Materno Perinatal en Perú.
\end{abstract}

Palabras clave: Transmisión vertical de enfermedad infecciosa; VIH; Infecciones por VIH; Guía de práctica clínica; Zidovudina (fuente: DeCS BIREME).

\section{RESULTS OF THE IMPLEMENTATION OF THREE NATIONAL GUIDELINES FOR THE PREVENTION OF HIV VERTICAL TRANSMISSION IN INSTITUTO NACIONAL MATERNO PERINATAL. LIMA, PERU}

\begin{abstract}
A retrospective analysis is performed in three successive periods between the years 1996 and 2009, in order to evaluate the impact of the implementation of three national guidelines for the prevention of the vertical transmission of HIV. 275 births were included in 13 years. Significant statistical differences were found in the percentage of HIV cases in the children exposed to the virus between the three periods: $15 \%$ during the period in which only zidovudine (AZT) was administered to the pregnant woman, 6.4\% during the second period (administration of AZT to the pregnant woman not fulfilling HAART initiation criteria and HAART to those fulfilling criteria for this treatment), and $4 \%$ during the third period in which HAART was applied to all pregnant women with HIV infection. 95\% of pregnant women ended their pregnancy by cesarean section and the $100 \%$ of children received infant formula. Changes made in national guidelines have produced a positive impact in the decrease of HIV infected children in the Instituto Nacional Materno Perinatal in Peru.
\end{abstract}

Key words: Infectious disease transmission, vertical; HIV; HIV infections; Practice guideline; Zidovudine (source: MeSH NLM).

\section{INTRODUCCIÓN}

A lo largo de los 30 años de epidemia del VIH/SIDA en el mundo, se han logrado muchos avances en el conocimiento y manejo de la infección. Uno de los acontecimientos históricos de mayor importancia sucedió en 1994, cuando se desarrolla el primer tratamiento en gestantes para disminuir la transmisión de madre a niño (transmisión vertical del VIH), basado en el uso de zidovudina como único antirretroviral ${ }^{(1,2)}$. Desde esa época, el conocimiento de nuevos antirretrovirales (ARV) que pueden ser utilizados en las gestantes sin producir daño al producto, ha permitido el uso de terapia antirretroviral de gran actividad (TARGA) y logrado disminuir significativamente el número de nacimientos de niños infectados, en los países donde se usa dicha terapia ${ }^{(3,4)}$.

En el Perú, el uso de ARV en gestantes se inició en 1996 como parte de la política nacional de lucha contra el SIDA; como tratamiento profiláctico en la gestante se usó zidovudina oral desde las 14 semanas, además de la cesárea programada y la suspensión de la lactancia materna, según la Directiva 002-96-PMP-PROCETSS,

Departamento de Neonatología, Instituto Nacional Materno Perinatal. Lima, Perú

a Médico Pediatra

Recibido: 02-04-11 Aprobado: 10-08-11 
Tabla 1. Directivas y normas nacionales de atención de gestantes con infección VIH en Perú, 1996-2011.

\begin{tabular}{|c|c|c|c|}
\hline $\begin{array}{l}\text { Periodo de } \\
\text { aplicación }\end{array}$ & $1996-2005$ & $2005-2007^{(6)}$ & $2008-2011^{(7)}$ \\
\hline $\begin{array}{l}\text { Sustento } \\
\text { científico }\end{array}$ & $\begin{array}{l}\text { Estudio ACTG } \\
076^{(2)}\end{array}$ & Recomendación de OMS ${ }^{(8)}$ & $\begin{array}{l}\text { Recomendaciones para el uso de ARV en } \\
\text { gestantes VIH para reducir la transmisión } \\
\text { perinatal del VIH en Estados Unidos }{ }^{(3)}\end{array}$ \\
\hline Esquema & $\begin{array}{l}\text { AZT desde las } \\
14 \text { semanas } \\
\text { de gestación }\end{array}$ & $\begin{array}{l}\text { Escenario 1: AZT en gestantes sin criterios } \\
\text { para inicio de TARGA } \\
\text { Escenario 2: TARGA en gestantes con } \\
\text { criterios de inicio de TARGA } \\
\text { Escenario 3: Continuar TARGA con las } \\
\text { gestantes que la reciben antes del embarazo } \\
\text { Escenario 4: Nevirapina en las gestantes } \\
\text { que se diagnostican por primera vez en el } \\
\text { momento del parto } \\
\text { Escenario 5: AZT en el niño cuya madre no } \\
\text { recibió tratamiento ARV }\end{array}$ & $\begin{array}{l}\text { Escenario 1: TARGA en gestantes con o sin } \\
\text { criterios de inicio } \\
\text { Escenario 2: continuar TARGA con las } \\
\text { gestantes que la reciben antes del embarazo } \\
\text { Escenario 3: AZT + Lamivudina + Nevirapina } \\
\text { en las gestantes que se diagnostican por } \\
\text { primera vez en el momento del parto } \\
\text { Escenario 4: AZT + Lamivudina en el niño } \\
\text { cuya madre no recibió tratamiento ARV }\end{array}$ \\
\hline
\end{tabular}

basada en el estudio ACTG076 (2), este protocolo ha tenido variaciones basadas en la evidencia y recomendaciones internacionales, conforme se describe en el Tabla 1.

A pesar de la experiencia adquirida en el manejo de la profilaxis para la transmisión vertical del $\mathrm{VIH}$, es difícil conocer el impacto que han tenido estos protocolos sobre la incidencia del VIH pediátrico en el país, debido a la ausencia de un sistema informático que permita registrar el seguimiento de los niños expuestos al VIH y de los infectados.

El Instituto Nacional Materno Perinatal (INMP) atiende aproximadamente 20000 partos por año y es considerada, por el número de nacimientos que atiende, como la tercera maternidad más grande en Latinoamérica. El INMP fue una de las primeras instituciones de salud en Latinoamérica, en aplicar el uso de ARV en gestantes para la prevención de la transmisión vertical del VIH desde 1996, como parte de una política de salud del Estado, y ha cumplido con aplicar los cambios de las guías nacionales hasta la actualidad. El trabajo realizado en el diagnóstico de las gestantes $\mathrm{VIH}$ y el seguimiento de los niños expuestos, hasta conocer su seroestado final, permite medir el impacto de la aplicación de estas guías sobre el número de nacidos infectados por el VIH.

\section{EL ESTUDIO}

Se empleó la base de registros de hijos nacidos de gestantes con infección por VIH, el año 1996 al 2009, se realizó el seguimiento de estos niños desde su nacimiento hasta su diagnóstico definitivo. Se incluyeron los registros en los que se indicaba el tratamiento materno y diagnóstico final del niño, considerando como niño sin infección por VIH si la prueba de ELISA para VIH era no reactiva a los 18 meses de edad y, a partir del 2005, si presentaba dos Pruebas de Reacción de Cadena Polimerasa (PCR) para VIH negativas hasta los seis meses edad. La prueba de ELISA usada para diagnóstico del niño fue procesada en el INMP y su confirmación (Western Blot, IFI), en el Instituto Nacional de Salud de Perú según lo establecido en las normas nacionales; las pruebas de PCR fueron procesadas e informadas por el Instituto Nacional de Salud de Perú. El estudio contó con la autorización institucional del INMP.

Los datos se dividieron en tres periodos, el primero entre 1996 a 2001, el segundo entre 2006 a 2007 y, el tercero, entre 2008 a 2009 de acuerdo con la vigencia de las guías nacionales sucesivamente desarrolladas por el Ministerio de Salud (5-7). Debido a limitaciones logísticas no se tuvo acceso a la información completa para el periodo 2002-2005 por lo que este periodo no se incluye en los resultados. Parte de la información correspondiente al primer periodo ha sido publicada en un estudio previo ${ }^{(9)}$.

Las diferencias de proporciones se midieron con la prueba estadística chi cuadrado. Se consideró como significativo un valor de $p<0,05$. El análisis se realizó con el paquete epidemiológico BioStat 2009.

\section{HALLAZGOS}

En total, de los 406 niños atendidos en el periodo de estudio, no se consideraron 131 registros por estar incompletos, 91 de ellos por falta de datos de algún parámetro de la profilaxis recibida por la madre y el niño (inicio de tratamiento, nombre de alguno de los ARV 
recibidos, tipo de nacimiento, profilaxis recibida por el niño); 32 por falta de información sobre el estatus final del producto y ocho registros por otras causas (no se confirmó infección materna). El número final de registros de nacimientos incluidos fue de 275 . El $95 \%$ de las gestantes VIH en el INMP culminaron el embarazo por cesárea y ninguno de los niños expuestos recibió lactancia materna.

En el primer periodo (1996 a 2001) se identificó a 196 hijos de madres con infección $\mathrm{VIH}$, de los cuales incluimos 120 niños $(61,2 \%)$ cuyas madres recibieron profilaxis con zidovudina o no recibieron ningún tratamiento. En este periodo, 18 niños (15\%) fueron positivos para VIH. De ellos, 5 (6,9\%) pertenecían al grupo de 72 niños cuyas madres recibieron tratamiento con AZT, y 13 (27,6\%) al grupo de 48 niños cuyas madres no recibieron tratamiento, existiendo diferencia estadística $(p=0,002)$.

En el segundo periodo (2006 a 2007) se identificó a 119 niños, de los que incluimos 78 (65,6 \%) niños cuyas madres recibieron profilaxis con zidovudina, con TARGA; o no recibieron ningún tratamiento. En el segundo periodo fueron $5(6,4 \%)$ los niños positivos para VIH; con dos niños $(3,1 \%)$ del grupo de 65 hijos de madres que recibieron algún tratamiento durante el embarazo; y tres (23\%) al grupo de trece que no recibieron tratamiento o lo hicieron solo en el parto, $(p=0,01$ para la diferencia de proporciones).

En el tercer periodo (2008 a 2009) se identificó 110 niños, de los que incluimos a 77 niños (70 \%) cuyas madres recibieron TARGA o ningún tratamiento. En este periodo, tres niños (4\%) fueron positivos para VIH. Entre aquellos niños cuyas madres recibieron TARGA, no se infectó ninguno de ellos, mientras que en el grupo cuya madre no recibió ningún tratamiento, se infectaron dos

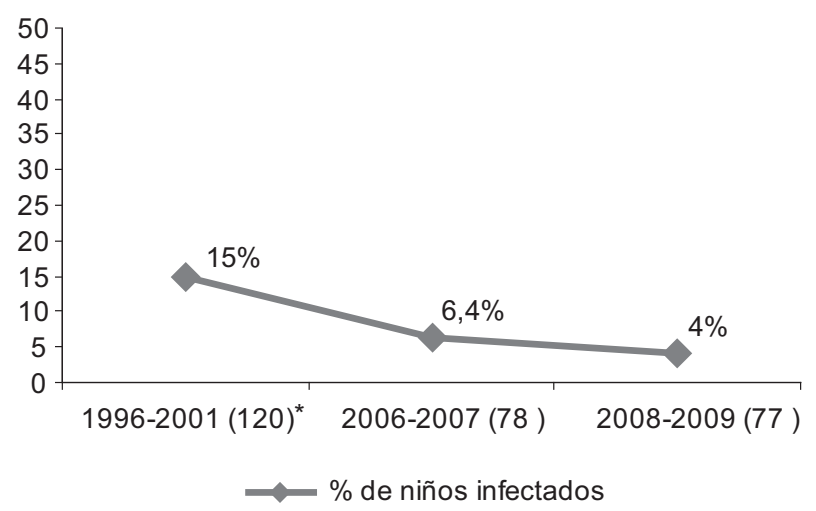

Figura 1. Porcentaje de niños infectados por el VIH nacidos en el Instituto Nacional Materno Perinatal de Perú, Periodo 19962009.

* Entre paréntesis, el tamaño de la población.

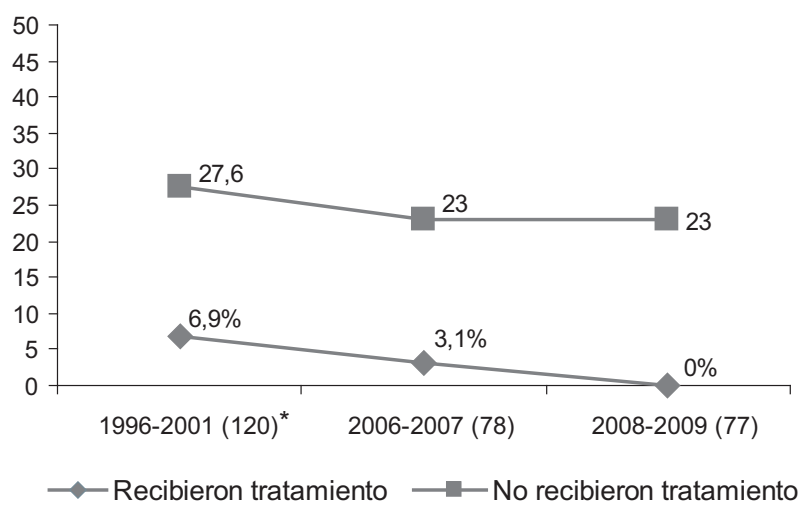

Figura 2. Comparación del porcentaje de niños infectados por el VIH según tratamiento recibido por su madre durante la gestación. Instituto Nacional Materno Perinatal de Perú, Periodo 1996-2009.

* Entre paréntesis, el tamaño de la población.

niños y en el que solo la recibieron en el momento del parto se infecto un niño, representando ambos grupos el $23 \%$ de los niños cuya madre no recibió TARGA durante el embarazo ( $p<0,001)$ (Figuras 1 y 2 ).

Se observa que a lo largo de los tres periodos de estudio, el total de niños nacidos con infección VIH en el INMP disminuyó significativamente de $15 \%$ a $4 \%$ $(p=0,0025)$.

\section{DISCUSIÓN}

Desde 1996 se han aplicado tres guías terapéuticas para la prevención de la transmisión vertical del VIH. Estas guías se diferenciaron en los siguientes esquemas: uno que incluía zidovudina como única droga durante la gestación; otro con zidovudina y TARGA, dependiendo si tenían o no criterios para el inicio la terapia de gran actividad; y finalmente al TARGA como único esquema de tratamiento durante el embarazo, protocolo que se usa actualmente.

En los resultados, se observa una clara tendencia a la disminución de la transmisión vertical del VIH desde 1996 a 2009, asociada inicialmente con el uso de ARV y mejorada tras la introducción del TARGA, lo cual disminuyó significativamente el total de niños infectados de 15 a $4 \%$.

El beneficio de los ARV se hace más evidente cuando se comparan a las poblaciones de gestantes $\mathrm{VIH}$ recibiendo este tratamiento, con aquellas que no lo recibieron en cada periodo; mientras que la infección por $\mathrm{VIH}$ se mantiene entre 23 y $27 \%$ en los grupos que no recibieron tratamiento los porcentajes disminuyeron de 6,9 a $0 \%$ en los grupos que sí recibieron tratamiento. 
Existen varios factores que pueden haber afectado los resultados, como la mayor cobertura de tratamiento ARV iniciado en el año 2005 en el Perú, las consejerías realizadas para lograr la menor pérdida de controles médicos durante la gestación y posteriormente de los niños, concientizando a las gestantes durante el control prenatal y realizando un tamizaje oportuno; además del mayor conocimiento en la población sobre el VIH en los últimos cinco años ${ }^{(10)}$, los menores problemas de abastecimiento de insumos para el diagnóstico, entre otras variables potencialmente confusoras, que requieren de un mayor análisis para medir su impacto en los resultados obtenidos. Sin embargo, la población atendida en el Instituto Nacional Materno Perinatal no ha variado su perfil en los últimos quince años. Un $30 \%$ acude a control prenatal en el primer trimestre del embarazo, $40 \%$ en el segundo y $30 \%$ en el tercero ${ }^{(11)}$. El promedio anual de gestantes $\mathrm{VIH}$ diagnosticadas en el INMP varía entre 50 y 60 al año, y las características socioeconómicas de las mujeres que viven con $\mathrm{VIH}$ no difieren del resto de la población que acude al Instituto ${ }^{(11)}$.

El periodo del embarazo en el cual se administran los ARV es también importante. Kourtis et al ${ }^{(12)}$ mediante un modelo matemático, establecieron una curva de distribución del riesgo de transmisión $\mathrm{VIH}$ de la madre a su niño de acuerdo con la etapa de la gestación en una población que no lacta, calculando que la mayor probabilidad de transmisión se encuentra a partir de las 36 semanas de gestación, con aproximadamente un $50 \%$ de riesgo, a comparación del primer trimestre, cuyo riesgo está alrededor del $3 \%$. Aunque en el presente estudio no se diferencia el momento de la gestación en que se inició el tratamiento antirretroviral, el mayor porcentaje de pacientes recibieron los ARV durante el segundo y tercer trimestre, los cuales son los periodos de gestación en los cuales se presentan con mayor frecuencia las gestantes a controlarse en nuestro Instituto (11), lo que podría explicar los resultados obtenidos.

Actualmente, está confirmado que el uso de combinaciones de ARV es más efectivo que el uso de una o dos drogas. El uso de TARGA durante la gestación logra disminuir hasta menos del $2 \%$ la posibilidad de infección en el niño ${ }^{(13)}$. Nuestro estudio demuestra este hecho cuando se comparan los tres periodos, la disminución de niños infectados se hace más evidente en el protocolo de TARGA para todas las gestantes que en aquellas poblaciones que recibieron los otros esquemas. En la población que no recibió tratamiento antirretroviral el riesgo de infección se encontró entre 23 y $27 \%$, similar a la literatura mundial ${ }^{(2-4)}$. Esto es de suma importancia puesto que muestra que, incluso en sistemas de salud subóptimos con poblaciones de escasos recursos y bajo nivel educativo, este tipo de intervenciones tienen alto impacto y deben de continuar usándose, además de ampliar su cobertura.

Como limitaciones del estudio, mencionaremos que no fue posible evaluar el efecto de la culminación del embarazo por cesárea y la suspensión de la lactancia materna, pues el $95 \%$ de mujeres diagnosticadas como portadoras de $\mathrm{VIH}$ durante el embarazo en el INMP culminan su gestación por cesárea y el $100 \%$ de niños expuestos no reciben lactancia materna. Además, la información recolectada en la base de datos se vio limitada por la falta de referencias en algunas historias maternas y de sus niños, lo que hizo que fueran descartadas, esto se produce sobre todo en el primer periodo, donde el sistema de seguimiento aún no se encontraba completamente implementado, lo que incluso motivó que no se pudiera analizar el periodo 2002-2005. Muchas de las pérdidas observadas se explican al cambio de hospitales o a la negativa que tenían algunas madres $\mathrm{VIH}$ que buscaban no ser identificadas y discriminadas al saber su estado tanto por el personal de salud, como por vecinos o conocidos. Por lo tanto, los resultados pueden verse limitados a las características propias de las pacientes que acuden al INMP, así como a la tecnología usada en el diagnóstico de la madre y el niño además de su registro.

En conclusión, nuestros resultados muestran que la implementación de las guías nacionales para tratamiento antirretroviral $y$, en particular, el uso de TARGA en gestantes infectadas, ha logrado una disminución significativa de la transmisión vertical del VIH en el Instituto Nacional Materno Perinatal

\section{Fuentes de financiamiento}

Autofinanciado.

\section{Conflictos de interés}

El autor declara no tener conflictos de interés en la publicación de este artículo.

\section{REFERENCIAS BIBLIOGRÁFICAS}

1. Connor EM, Sperling RS, Gelber R, Kiselev P, Scott G, O'Sullivan MJ, et al. Reduction of maternal-infant transmission of human immunodeficiency virus type 1 with zidovudine treatment. Pediatric AIDS Clinical Trials Group Protocol 076 Study Group. N Engl J Med. 1994;331(18):1173-80.

2. Sperling RS, Shapiro DE, Coombs RW, Todd JA, Herman SA, McSherry GD, et al. Maternal viral load, zidovudine treatment, and the risk of transmission of human immunodeficiency virus type 1 from mother to infant. Pediatric AIDS Clinical Trials Group Protocol 076 Study Group. N Engl J Med. 1996;335(22):1621-9. 
3. US Department of Health and Human Services. Recommendations for Use of Antiretroviral Drugs in Pregnant HIV1-Infected Women for Maternal Health and Interventions to Reduce Perinatal HIV Transmission in the United States. Washington, DC: HHS; 2007. Disponible en: http://www. uphs.upenn.edu/bugdrug/antibiotic_ma

4. US Department of Health and Human Services. Recommendations for Use of Antiretroviral Drugs in Pregnant HIV1-Infected Women for Maternal Health and Interventions to Reduce Perinatal HIV Transmission in the United States. Washington, DC: HHS; 2010. Disponible en: http://www. aidsinfo.nih.gov/ContentFiles/PerinatalGL.pdf

5. Ministerio de Salud del Perú. Disminución de la Transmisión Vertical del VIH y Manejo de la Gestante que Vive con VIH. Directiva Conjunta N 002-98-PMP - PROCETSS. Programa de Salud Materno Perinatal - PROCETSS. Lima: MINSA; 1998.

6. Ministerio de Salud del Perú. Norma Técnica para la Prevención de la Transmisión Vertical (madre-niño) del VIH. NT 024-2005-MINSA/DGSP-V01. Lima: MINSA; 2005.

7. Ministerio de Salud del Perú. Norma Técnica de Salud para la Profilaxis de la Transmisión madre-niño del VIH y la Sífilis Congénita. NTS Nº 064-2008-MINSA/DGSP-V01. Lima: MINSA; 2008.

8. WHO. Antiretroviral Drugs for Treating Pregnant Women and Preventing HIV Infection in Infants. Ginebra: WHO; 2004.
9. Velásquez C. Transmisión Vertical del VIH-1. Instituto Materno Perinatal de Lima. Ginecol Obstet (Perú). 2002;48(4):235-42.

10. Instituto Nacional de Estadística e Informática. Encuesta Demográfica y de Salud Familiar. ENDES Continua 2010. Lima: INEI; 2010 [Citado: Agosto 2011]. Disponible en http://proyectos.inei.gob.pe/endes/endes2010/resultados/index.html.

11. Instituto Nacional Materno Perinatal. Plan Estratégico Institucional 2008-2011. Lima: INMP; 2008.

12. Kourtis A, Lee F, Abrams E. Mother-to-child transmission of HIV-1: timing and implications for prevention. Lancet. 2006;6:726-32.

13. Cooper ER, Charurat M, Mofenson L, Hanson IC, Pitt J, Diaz C, et al. Combination antiretroviral strategies for the treatment of pregnant HIV-1 infected women and prevention of perinatal HIV-1 transmission. J Acquir Immune Defic Syndr. 2002;29(5):484-94.

Correspondencia: Carlos Velásquez Vásquez Dirección: Jr. Miroquesada 941. Lima, Perú. Teléfono: 328-0998 anexo 1136

Correo electrónico: Carlos.carvel@gmail.com

\section{Consulte las ediciones anteriores de la} Revista Peruana de Medicina Experimental y Salud Pública en

\section{www.scielosp.org}

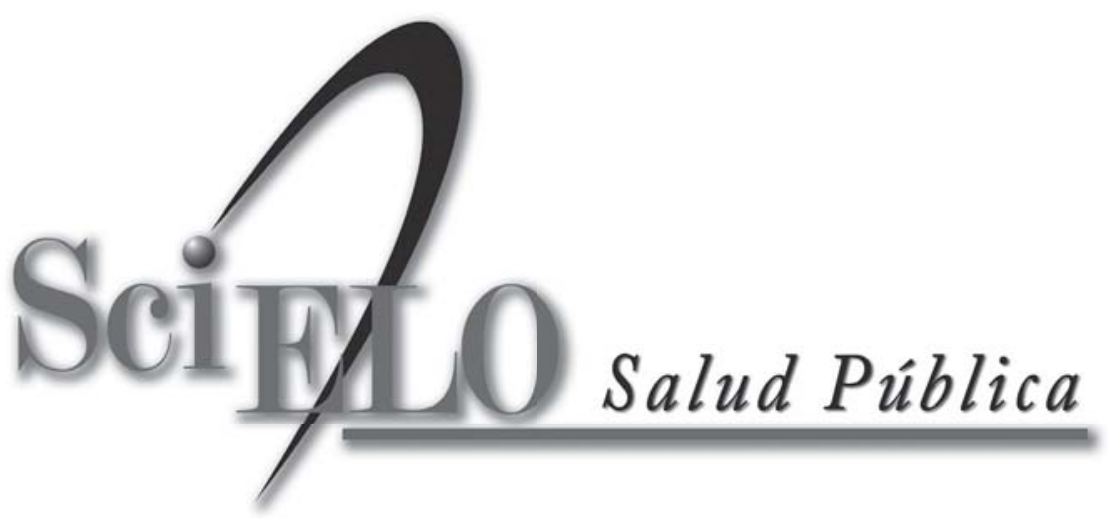

